\title{
Retrieving and Connecting Reputations to Find Reliable Results
}

\author{
Somayeh Khatiban \\ City University London \\ School of Informatics \\ Somayeh.khatiban.1@city.ac.uk
}

\begin{abstract}
Online reputation addresses trust relationship amongst agents in the dynamic open systems which can appear as ratings, recommendations, referrals and feedbacks. Several reputation models and rating aggregation algorithms have been proposed. However, finding a trusted object on the web is still an issue as all reputation systems work individually. The aim of this project is to introduce a reputation system that takes advantage of different types of reputation data over the web to make a trusted choice as a whole in the search result.
\end{abstract}

Reputation system, information retrieval, social media

\section{INTRODUCTION}

Reputation has been defined as the "opinion or view of one about something" [1]. Reputation is also known as rating, recommendations, referrals, and feedbacks [2]. A reputation system collects these opinions over the time and will develop behaviour of an agent in the system. As a result of this system the user can make a decision to choose a product, service or an expert based on the recommendations of other users. Rating and feedbacks supply an effective way to create a trust relationship among similar objects in open environments [2]. Many online marketplaces have their own ranking and review systems in order to help shoppers to make a better choice in terms of relevancy, price, and other factors. Moreover, business directories offer a platform for businesses to list their products and services to make it easier for users to find them. Often they supply a rating system for users to leave their feedbacks as well. However, the results of reputation systems are usually accessible (and sometimes meaningful) within the system providers; whereas they are valuable resources which can improve the trust in the search result. This rating content is often useful and relevant to the user's information need but, they are dispersed over the web and there is no connection between them.

A comparison between reputation information will help to find a link among available ratings on an object in various systems. Mui, et al [3] studied reputation in various disciplines and proposed a typology to unify different notion of reputation. They discussed the qualitative comparison in the diverse literature on reputation and introduced a simulation framework to compare them quantitatively. Furthermore, Morris, et al. [4] looked into question asking behaviour on social networking services by a survey and they reported the motivation of people for using these services. They identified two methods of search using social networks. First, social search which refers asking questions from people in the network such as question-andanswering (Q\&A) tools. Second, collaborative search which addresses sharing information with others.

Reputation and social behaviour can be investigated form another perspective. Imagine that you are planning to buy an item. You are willing to know what other users said about this item and what are reviews and feedbacks. You may consider social media, Q\&A sources, online marketplaces and business directories that provide ranking and review systems to find out about reputation of the item. Thus, you will face to an overwhelming data which are spread over the web about that item. Furthermore, you have to compare your findings depending on the algorithm, scale and measurement that they use which is a hard work with a high margin of error.

Diaz et al. [5] defined federated search an information retrieval technique that search multiple resources rather than a single centralised retrieval system. They compared it with aggregated search which refers to the combination of non-web content such as images, news articles and so on. They pictured how federated search is effective to query from several source of data and how aggregated search can put the result together in a standard format. Among this aggregated data some of them 
are pointing reputation from various perspectives. However, even though they are addressing the same nature of data, there is no connection between them to compare them and summarise them.

The given scenario leads to the following research questions:

- Find a connection between reputation elements about an item in various systems

- Find similarities between reputations to compare them

\section{RELATED WORK}

Finding information based on reputation and trust can be addressed from different viewpoints, including reputation systems, which discuss different models and important factors of reputations, and recommendation systems which studies personalisation. Besides, social networks and Q\&A systems have also been used for social search. Finally collaborative information retrieval and expert finding methods cover social approaches for information finding.

Recommendation systems are being used to personalise user's profile. The recommendation algorithm for Amazon marketplace is based on Item-to-Item Collaborative Filtering [6]. It compares purchased items with other items rather than customer profiles which will give a high quality recommendation with less online computation.

Besiki and Jörgensen [7] discussed the structure and pattern of activities. They scaled and analysed the quality of generated metadata in a social media (Flickr).

This work involves rank aggregation or combining many sources to produce a ranking of documents [8]. The reputation system that is the aim of this project involves aggregating social network activities and generated data from other individual reputation systems. One of the related works in aggregation is WaterCooler tool [9] which collects internal social media activities in HP Company and cross-references them with the organisation's directory. This helps the company to find people with the right expertise or interest as well as finding needed information. In addition, it motivates employees to share their knowledge.

The user-oriented model for expert finding [10] takes two constraints for finding an expert in a test collection: time to contact a person, and the knowledge of the person about the topic. Both [10] and [4] have evidences that there is a relation between the answer time and the quality of the answer.

\section{RESEARCH METHODOLOGY}

Figure 1 illustrates the current state of reputation systems. In the figure 2 the possible solution has been pictured.
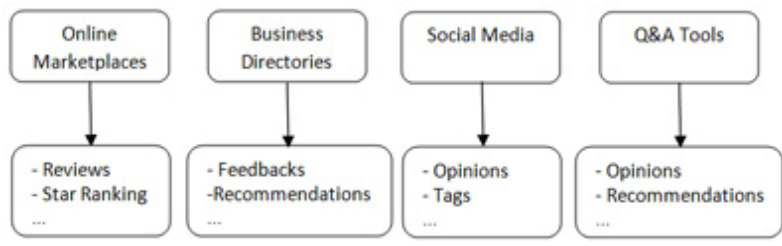

Figure 1: Reputation derive from different source of data

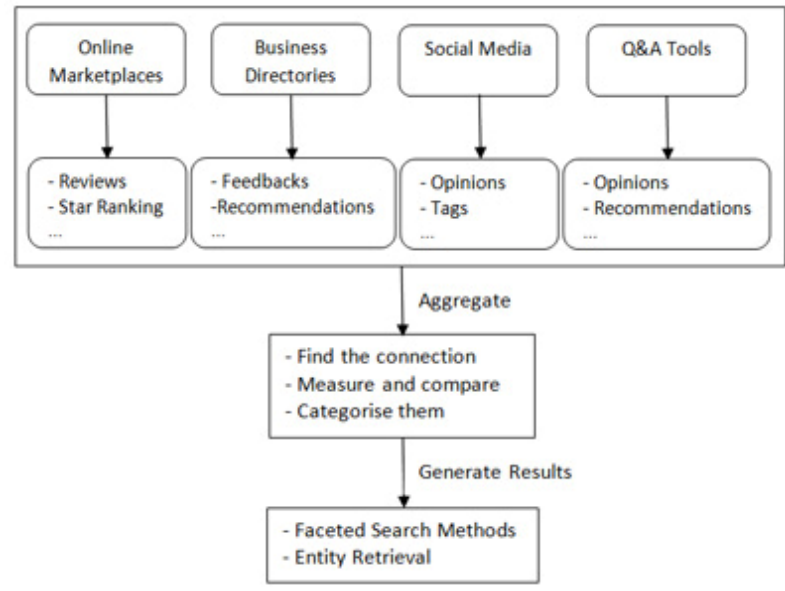

Figure 2: Finding connection between reputation data and find a link between them (first research question) and finding similarities (second research question)

This work will involve a study on reputation and ranking data to find reputation data characteristics. This will enable us to find similarities amongst them and will make it possible to compare them. Then they can be assigned to multiple classifications and be presented using faceted search and entity retrieval techniques in the result.

\section{CONCLUSION}

In this work, we propose the task of combining different types of reputations and finding a connection between them. This can lead us to generate more relevant and appropriate results using faceted search and entity retrieval methods. In the future this can feed metadata search as well.

\section{REFERENCES}

[1] J. Sabater, and C. Sierra, REGRET: reputation in gregarious societies, Proceedings of the fifth international conference on Autonomous agents, 
ACM, Montreal, Quebec, Canada, 2001, pp. 194195.

[2] Zhengqiang Liang, Weisong Shi. (2005). Performance Evaluation of Rating Aggregation Algorithms in Reputation Systems. IEEE COLCOM, page $10 \mathrm{pp}$.

[3] L. Mui, M. Mohtashemi, and A. Halberstadt, Notions of reputation in multi-agents systems: a review, Proceedings of the first international joint conference on Autonomous agents and multiagent systems: part 1, ACM, Bologna, Italy, 2002, pp. 280-287.

[4] M. Morris, J. Teevan, and K. Panovich, What do people ask their social networks, and why?: a survey study of status message Q\&A behavior, Proceedings of the 28th international conference on Human factors in computing systems, ACM, Atlanta, Georgia, USA, 2010, pp. 1739-1748.

[5] F. Diaz, M. Lalmas, and M. Shokouhi, From federated to aggregated search, Proceeding of the 33rd international ACM SIGIR conference on Research and development in information retrieval, ACM, Geneva, Switzerland, 2010, pp. 910-910.

[6] Greg Linden, Brent Smith, and Jeremy York. (2003). Amazon.com Recommendations Item-toItem Collaborative Filtering. IEEE Data Engineering Bulletin, 76-80

[7] Besiki Stvilia and Corinne Jörgensen. (2010). Member Activities and Quality of Tags in a Collection of Historical Photographs in Flickr. JASIST 61 (12) 2477-2489.

[8] M. Farah, and D. Vanderpooten, An outranking approach for rank aggregation in information retrieval, Proceedings of the 30th annual international ACM SIGIR conference on Research and development in information retrieval, ACM, Amsterdam, The Netherlands, 2007, pp. 591-598.

[9] Brzozowski Michael, WaterCooler: exploring an organization through enterprise social media, Proceedings of the ACM 2009 international conference on Supporting group work, ACM, Sanibel Island, Florida, USA, 2009, pp. 219-228.

[10] E. Smirnova, and K. Balog, A user-oriented model for expert finding, Proceedings of the 33rd European conference on Advances in information retrieval, Springer-Verlag, Dublin, Ireland, 2011, pp. 580-592. 